\title{
Analysis on the detection rate of Giardia lamblia in children of Sichuan province of China
}

\author{
Zhengqiang Hu \\ Sichuan University \\ Yonglin Zhong \\ Sichuan University \\ Jie Li \\ Sichuan University \\ Yunxia Li ( $\nabla 1545807456 @ q q . c o m$ ) \\ Sichuan University
}

\section{Research Article}

Keywords: Giardia lamblia, Detection rate, Children, Ethnic minority, Fecal examination

Posted Date: February 22nd, 2022

DOI: https://doi.org/10.21203/rs.3.rs-1254278/v2

License: (9) This work is licensed under a Creative Commons Attribution 4.0 International License. Read Full License 


\section{Abstract \\ Objective}

To investigate the detection rates of Giardia lamblia in children of Sichuan province and analyze the susceptible population and the cause of infection.

\section{Methods}

The routine fecal examination of a total of 11575 cases were done by normal wet smear microscopy, and the suspected cases were stained with iodine in outpatient children of West China Second University Hospital, Sichuan University from September 1st, 2020 to August 31th, 2021.

\section{Results}

In the stools of 11,515 outpatient children, 11 cases of cysts or trophozoites of $G$. lamblia were detected, among which 9 cases were from ethnic minority patients and 2 case were from Han patients. The $G$. lamblia detection rate of Han patients was $0.019 \%$, while that of ethnic minority patients was $0.968 \%$, between which there was a statistically significant difference $(P<0.05)$.

\section{Conclusions}

The detection rate of $G$. lamblia in minority patients was higher than that in Han patients. To reduce the spread and epidemic of giardiasis, the epidemic prevention in minority areas should be strengthened and the good hygienic habits of children should be cultivated.

\section{Introduction}

Giardia lamblia, also known as Giardia duodenalis, is one of the most susceptible intestinal parasitic protozoa in children[1]. G. Iamblia parasitizes the human small intestine, gallbladder, mainly in the duodenum, causing abdominal pain, diarrhea, vomiting and indigestion-based symptoms, called giardiasis[2-3]. G. lamblia is a flagellate that does not invade epithelial cells and reproduces asexual only by binary division. They produce resistance stages (Giardia cysts) and are released into the environment as a mode of infection for these intestinal parasitic protozoa[3]. G. lamblia exhibit a biphasic lifestyle, i.e. dormant cysts or reproductive trophozoites, which propagate by the dichotomy of the trophozoites. Cysts can be found in stools with normal hardness, and trophozoites may be found in cases of diarrhea. At the same time, $G$. lamblia is a zoonotic intestinal parasitic protozoa that may be transmitted from humans to animals[4-5]. 
G. lamblia is one of the most prevalent parasite infections in the world. It is also a common intestinal protozoa that infects both humans and animals [6]. About 200 million people worldwide are infected with G. lamblia every year, with a high incidence among tourists. So, diarrhea caused by G. lamblia is also called "tourist diarrhea"[7]. The survey of G. lamblia infection status in 11 provinces (or municipalities and autonomous regions) of China shows that the infection rate is $0-16.2 \%$, of which the infection rate of HIV patients and immunodeficiency populations is higher[8-9]. However, the ethnic distribution of giardiasis in Sichuan province of China and the difference in detection rates of different ethnic groups have not been reported. This study was to carry out routine fecal examinations for outpatient children in our hospital and investigate the detection rates of giardiasis in Sichuan province, and to analyze the susceptible people and susceptible causation of this disease.

\section{Materials And Methods}

\section{Patients}

A total of 11575 cases of outpatient children aged 0- to 12-year-old (mean $\pm S D 6.85 \pm 4.41$ years) in West China Second University Hospital, Sichuan University from September 1st, 2020 to August 31th, 2021 were enrolled in this study. Inclusion criteria were as follow: 1) Informed consents were acquired from patients and their guardians, and patients were from 0- to 12-year-old; 2) Patients who had clinical symptoms of abdominal pain, diarrhea, vomiting and indigestion-based symptoms, etc.; 3) Outpatient children with recent diagnosis in our hospital and who were not previously treated with anti-infective drugs for intestinal tract. Exclusion criteria were as follow: 1) Patients who were treated with anti-infective agents for intestinal tract, or with other agents that would cause interference for diagnosis; 2) Patients who were over 12-year-old.

\section{Methods}

The routine fecal examination of a total of 11575 cases were done by normal wet smear microscopy, and the suspected cases were stained with iodine. Among which, there were 10,645 Han patients (mean \pm SD $6.91 \pm 4.68$ years) and 930 ethnic minority patients(mean \pm SD $5.96 \pm 4.05$ years). The found of Giardium cysts or trophozoites were diagnosed as positive giardiasis cases(Figure 1). And then, the nationality, month of birth, living environment, hygiene habits, current medical history, and previous history of all patients were investigated. In our study, the written informed consent was obtained from all participants and the privacy rights of them were also reserved. And all procedures and protocols are in accordance with the Helsinki Declaration as revised in 2013.The study protocol(Medical Research 2020, No.40)was approved by the Institutional Review Board(IRB) of the West China Second University Hospital, Sichuan University on 26th July 2020 before study initiation.

\section{Statistical analysis}

The database was established using Excel sheet to calculate the positive rate of giardiasis. The positive proportion of Han and minority outpatient children was calculated using the $\chi^{2}$ test correction formula. 
$P<0.05$ was considered statistically significant[10].

\section{Results}

In the stools of 11,515 outpatient children, 11 cases of cysts or trophozoites of $G$. lamblia were detected, among which 9 cases were of ethnic minority patients and 2 case was of Han patients. Among the 9 ethnic minority giardiasis patients, 6 were Tibetans and 3 were Yi nationalities. The total G. lamblia detection rate was $0.095 \%$. The detection rate of G. lamblia of Han patients was $0.019 \%$, meanwhile that of the ethnic minority patients was $0.968 \%$ (Table 1 ), between which there was a statistically significant difference $\left(X^{2}=81.12, P<0.05\right)$. The detection rates of $G$. lamblia in children of different nationalities were shown in Table 2.

Table 1

The detection rates of $G$. Iamblia in 11,575 children of Sichuan province

\begin{tabular}{|llllll|}
\hline Nationality & $\mathbf{n 1}$ (total) & n2(detected) & Detection rates(\%) & $\boldsymbol{\chi}^{2}$ & $\boldsymbol{P}$ \\
\hline Han nationality & 10645 & 2 & 0.019 & 81.12 & $<0.05$ \\
Ethnic minority & 930 & 9 & 0.968 & & \\
\hline Total & 11575 & 11 & 0.095 & & \\
\hline
\end{tabular}

Table 2

The detection rates of G. lamblia in children of different nationalities of Sichuan province

\begin{tabular}{|llll|}
\hline Nationality & N1(total) & N2(detected) & Detection rates(\%) \\
\hline Han nationality & 10645 & 2 & 0.019 \\
\hline Tibetan & 446 & 6 & 1.345 \\
\hline Yi nationality & 263 & 3 & 1.141 \\
\hline Miao nationality & 33 & 0 & 0 \\
\hline Hui nationality & 32 & 0 & 0 \\
\hline Qiang nationality & 30 & 0 & 0 \\
\hline Other nationality & 126 & 0 & 0 \\
\hline Total & 11575 & 11 & 0.095 \\
\hline $\begin{array}{l}\text { Note: Due to the large ethnic minorities of the outpatient children were included, the top six ethnic } \\
\text { minorities of them were included in the statistics, and the number of outpatient children with other } \\
\text { ethnic minorities was combined. }\end{array}$ \\
\hline
\end{tabular}

\section{Discussion}


G. lamblia is a common intestinal parasitic protozoa associated with diarrhoea disease, and also a zoonotic parasite divided into five genotype assemblages of A-E. Studies have shown that the infection rate of G. lamblia of patients with HIV and the immunodeficiency population is higher [7-8].At the same time, the research results showed that $G$. lamblia infection existed in HIV/AIDS patients in Guangxi, and the genotype was mainly assemblage $B$, and host-specific assemblage $C$ was found[5]. Genotype of $G$. lamblia isolated from different hosts suggests that the host species with the largest infection range of assemblages $A$ and $B$, appears to be the primary (or possibly only)assemblage of $G$. lamblia. At least in some cases of wild mammalian assemblage $A$ or $B$ infection, there is evidence that the infection was caused by environmental contamination from the $G$. lamblia cysts of human origin[11].

The analytic method of $G$. lamblia directly related to its sensitivity, specificity and detection rate. Currently, G. lamblia assays include direct microscopic, staining, immunological methods and molecular diagnosis. The traditional microscopy method is the "gold standard" for $G$. lamblia detection. However, it is timeconsuming and low detection rate[12]. Immunological methods, such as immuno-fluorescence, ELISA, have improved sensitivity and specificity compared with traditional microscopy. But the former cannot distinguish insect species, the latter is prone to cross-reactions, all of them cannot accurately quantify the number of G. lamblia, and were used poorly in mildly infected fecal samples[13]. With high sensitivity and good specificity, the fluorescent quantitation PCR (FQ-PCR) method, which is one of the preferred methods for the quantitative detection of $G$. lamblia, can detect a large number of samples simultaneously[14]. In recent years, isothermal nucleic acid amplification technology has been widely used in the field of prevention and control of infectious disease, mainly including chain replacement amplification, recombinase polymerase amplification technology and loop-mediated isothermal amplification (LAMP).The LAMP technology has a high degree of specificity and sensitivity, good stability and repetitive in the gene sequence analysis of triose phosphate isomerase (TPI) of G. lamblia [15].

In our study, 9 of the 11 giardiasis patients detected in our hospital were ethnic minority children (6 Tibetans and 3 Yi nationalities), and 2 was Han patients. The detection rate of $G$. lamblia in minority patients is higher than that in Han patients. Meanwhile, one of the Han patients had a life history in $\mathrm{Yi}$ nationality area and $G$. lamblia have not been detected in other minority children. Considering the intermittent nature of the discharge of the stools and the inexperience of the inspectors, the detection rate of $G$. lamblia in Sichuan Province may be higher [16]. Through analyzing their life history and susceptible reasons, the pathogeny of giardiasis was related to health conditions, economic status, cultural level, unclean eating habits and living habits in ethnic minority areas, as well as related to the animal husbandry that the local residents mainly engaged in[17]. G. lamblia has a simple life history and spreads extremely fast. People are infected mainly by ingestion of water and food contaminated with cysts. Improper management of human and animal stools, without washing hands before and after meals, and lack of awareness of epidemic prevention constitute important risk factors for $G$. lamblia infection.

The parents of children work outside, leading to the patients stay in the rural areas under the care of other generations of elders. Due to poor care, the patients may have no personal health position. The children of Kindergarten, nursery, and primary school live together, and play toys with each other can also cause 
potential risk factors spreading from person to person [18]. 9 outpatient children with giardiasis were less than 5 years old in our hospital, 2 patients was aged over 5 years (12-year-old), which suggests that children under 5-year-old are more susceptible to Giarasis. Mahdy, et al. have identified children under 12year-old as populations at high risk for $G$. lamblia infection[19]. Han, et al. suggested that children (0- to 4-years-old) were 17.911 times more susceptible than adults (15- to 64-year- old)[20].All these suggest that children are more susceptible to giardiasis.

The outpatient children in our hospital are mainly derived from all over Sichuan Province, and many kinds of intestinal protozoa and sporozoa have been detected in their stools. Children with giardiasis often intermittently discharge cysts or trophozoites, which are prone to repeated infection. It is suggested that the primary hospitals should conduct regular census and repeated screening of the population. Insect repellent measures should be taken immediately if the confirmed cases are found. Health and epidemic prevention departments should strengthen the fecal management of human and animal hosts, do a good job in environmental sanitation, and prevent water being polluted. Children should be cultivated to develop good personal hygiene and living habits, and reduce the risk of fecal-hand-mouth-oral transmission and human and animal transmission. At the same time, it is recommended that the local Center for Disease Control and Prevention(CDC) publicize and popularize the relevant knowledge of parasitic diseases to children and their parents in remote areas. For inspectors, we should improve our professional ability and detection experience to reduce the missed detection rate. Immunology and molecular diagnostic techniques based on traditional microscopy should be increased to improve the sensitivity and specificity of $G$. lamblia detection. It is suggested to strengthen the professional training and assessment of the relevant inspectors, and to further improve the detection rate and diagnosis rate of giardiasis.

\section{Conclusions}

There was high detection rate of $G$. lamblia in ethnic minority patients, which was mainly related to its personal hygiene, living habits and eating habits. It is suggested to strengthen the epidemic prevention and management in ethnic minority areas. And children's good hygiene habits should be cultivated, so as to reduce the spread and prevalence of giardiasis.

\section{Abbreviations}

\section{G. Lamblia}

Giardia lamblia

FQ-PCR

fluorescent quantitation PCR

LAMP

loop-mediated isothermal amplification

TPI

triose phosphate isomerase 
CDC

Center for Disease Control and Prevention.

\section{Declarations}

\section{Ethics approval and consent to participate}

All procedures performed in studies involving human participants were in accordance with the ethical standards of the institutional and/or national research committee and with the 1964 Helsinki declaration and its later amendments or comparable ethical standards.

\section{Consent for publication}

Informed consent was obtained from all individual participants included in the study.

\section{Competing interests}

All authors declares that he/she has no competing of interest.

\section{Funding}

This research received no specific grant from any funding agency in the public, commercial, or not-forprofit sectors.

\section{Authors' contributions}

Zhengqiang Hu drafted the manuscript. Zhengqiang Hu and Yonglin Zhong participated to acquisition of data. Jie Li generated the experimental results. Yunxia Li designed the study and reviewed the manuscript for intellectual content. All authors approved the final version of the manuscript.

Data curation: Zhengqiang Hu, Yonglin Zhong.

Formal analysis: Yonglin Zhong.

Project administration: Jie Li.

Writing-original draft: Zhengqiang $\mathrm{Hu}$.

Writing-review \& editing: Yunxia Li.

\section{Acknowledgements}

We would like to extend sincere gratitude and thanks to the staff of the Department of Laboratory Medicine, the Outpatient Department of Obstetrics and Gynecology of the West China Second University Hospital, Sichuan University, China, and the investigators of Key Laboratory of Birth Defects and Related Diseases of Women and Children (Sichuan University), Ministry of Education. 


\section{Availability of data and material}

All authors declares that the data and material were available.

\section{References}

[1] Yu YF. The infection of Giardia lamblia and it's genotype of HIV infected person and kindergarten children in rural areas of Anhui province. Chinese Journal of Parasitonology and Parasiosis, 2016, 34(6): 537-541. (in Chinese)

[2] Zhu MS, Zhu J, Wang SJ, et al. Investigation of infection of Giardia lamblia in children of Shiyan City. Health in Chinese schools, 2009, 30(12): 1136-1137. (in Chinese)

[3] Vitaliano AC, Blaine AM. Infections by Intestinal Coccidia and Giardia duodenalis. Clin Lab Med, 2015, 35(2): 423-444.

[4] Marc YF, Danielle S, Steven MS. Giardia lamblia: Laboratory Maintenance, Lifecycle Induction, and Infection of Murine Models. Curr Protoc Microbiol. 2020, 57(1): e102. doi:10.1002/cpmc.102.

[5] Liu H, Xu N, Shen YJ, et al. Infection and genotype of Giardia lamblia among HIV/AIDS patients in Guangxi

Region. Chinese Journal of Parasitonology and Parasiosis, 2019, 37(3): 321-325. (in Chinese)

[6] Berry,ASF.Johnson,K.Martins,R.Beiting,DP et al. Natural infection with Giardia is associated with

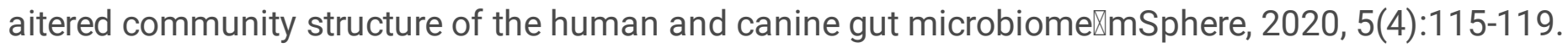

[7] Li J, Wang H, Wang R, et al. Giardia duodenalis infections in humans and other animals in china. Front Microbiol, 2017, 8: 2004.

[8] Teng XJ, Chen JX, Tian LG. The status of HIV/AIDS patients Combined intestinal protoplasma infection. Chinese Journal of Parasitonology and Parasiosis, 2017, 35(6): 607-614. (in Chinese)

[9] Wang L, Xia YT, Tian XH, et al. Analysis of infection and influencing factors of Giardia lamblia in people of part of southern China in 2014. The Journal of the Environment and Health, 2018, 35(3): 238241. (in Chinese)

[10] Li K,He J. Medical Statistics. (7th ed).Beijing: People's Medical Publishing House Co., Ltd., 2018, pp.14-20.

[11] Martin FH. Giardia duodenalis genetic assemblages and hosts. Parasite, 2016, 23(13):1-4.

[12] Elsafi SH囚Al-Maqati TNखHussein MI, et al. Comparison of microscopy, rapid immunoassay, and molecular techniques for the detection of Giardia lamblia and Cryptosporidium parvum. Parasitol Res, 112(4):1641-46. 
[13] Zhang XP, Zhu Q, Jiang SF, er al. The comparative study of Giardia lamblia and Cryptosporidium contamination by two applied detection methods in urban water sources $\ T$ The International Journal of Medical Parasiosis, 2015, 42(6):346-351. (in Chinese)

[14] Li J, Huang DN, Zhang XM, et al. The establishment of a two-step detection technique of double fluorescent quantitation PCR in Giardia lamblia and Cryptosporidium parvum. Chinese Journal of Zoorbidity, 2020, 36(1):32-39. (in Chinese)

[15] Yu MC, Yang ZW, Wang HR, et al. The establishment of a method for detecting Giardia lamblia based on LAMP microfluidic chip. Chinese Journal of Parasitonology and Parasiosis, 2021, 39(3): 402-405. (in Chinese)

[16] Chen RX, Zheng ZL. Analysis of parasite egg leakage in fecal examination. The Chinese Journal of Parasitic Disease Prevention and Control, 2004, 17(5): 278-278. (in Chinese)

[17] Hussein EM, Zaki WM, Ahmed SA, et al. Predominance of Giardia lamblia assemblage A among iron deficiency anaemic pre-school Egyptian children. Parasitology Research, 2016, 115(4): 1537-1545. DOI:10.1007/s00436-015-4888-y.

[18] Yu XG, Hu W, Li GQ. Preliminary exploration of potential pathogenic mechanism of Giardia lamblia. The International Journal of Medical Parasiosis, 2015, 42(2): 112-117. (in Chinese)

[19] Mohammed Mahdy AK. Lim YAL, Surin J, et al. Risk factors for endemic giardiasis: highlighting the possible association of contaminated water and food. Transactions of the Royal Society of Tropical Medicine and Hygiene, 2008, 102(5): 465-470. DOI: 10.1016/j.trstmh.2008.02.004.

[20] Han M, Xiao S, An W, Yang M, et al. Co-infection risk assessment of Giardia and Cryptosporidium with HIV considering synergistic effects and age sensitivity using disability-adjusted life years. Water Research, 2020, 175:115698. (in Chinese)

\section{Figures}




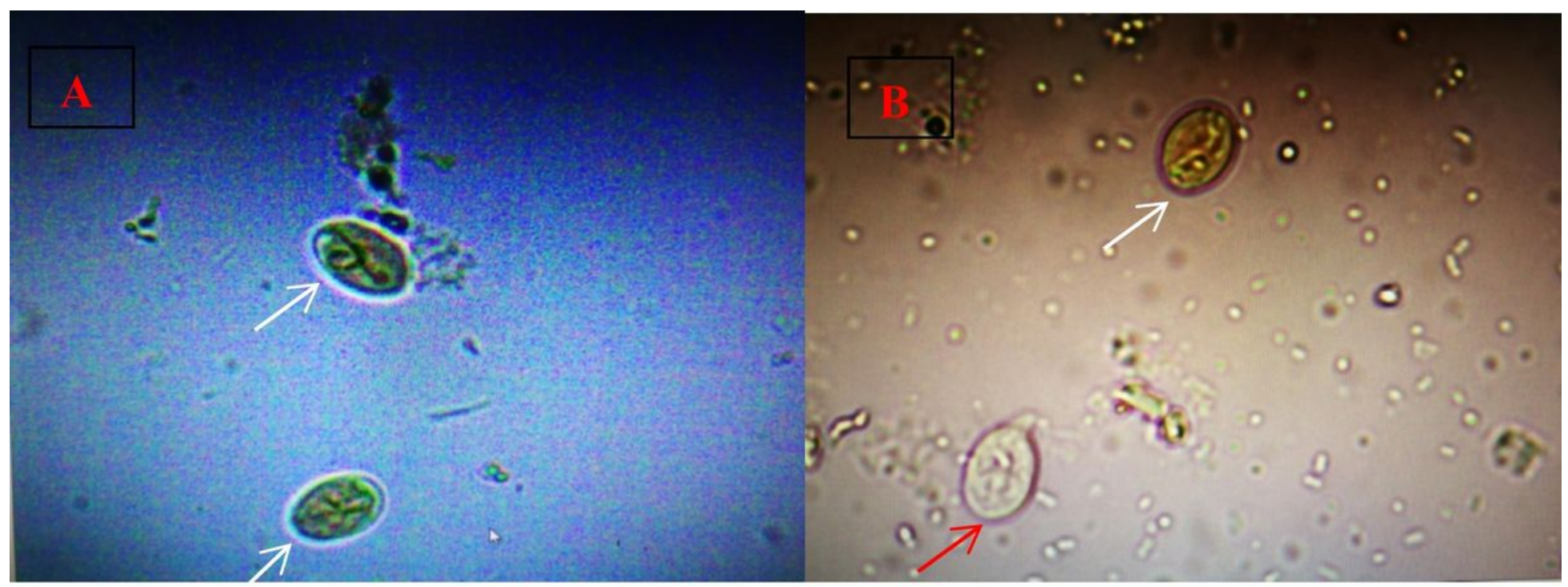

\section{Figure 1}

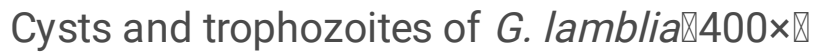

Note: Figure 1-A is the saline wet smear with fluorescent screen background; Figure 1-B is the iodinestained wet smear. White arrowheads indicate Giardia cysts, and red arrows show Giardia trophozoites.

\section{Supplementary Files}

This is a list of supplementary files associated with this preprint. Click to download.

- GraphicalAbstract.doc 